\title{
ACOUSTIC ENVIRONMENT RESULTING IN INTERACTION OF LAUNCH VEHICLE MAIN ENGINES JETS WITH A LAUNCH PAD HAVING CLOSED LONG DUCTS LIKE A TUNNEL
}

\author{
V. V. Kudryavtsev and A. V. Safronov \\ Central Research Institute of Machine Building (TSNIIMASH) \\ Pionerskaya Str. 4, Korolev 141070, Moscow region, Russia
}

\begin{abstract}
Paper deals with elaboration of semiempirical technique for prediction of broadband acoustic field generated at interaction of launch vehicle main engines jets with a launch pad having closed long ducts like a tunnel. Approach to a problem is based on analysis of jet interaction with typical deflectors, extraction of characteristic noise generation regions, and substitution of each region of noise generation by a system of independent acoustic sources with prescribed acoustic power and spectrum of acoustic radiation. Comparisons of calculated results with experimental data indicate that the technique allows to make reliable estimations of acoustical field characteristics as a function of geometrical and gasdynamic parameters and to analyze different means for reduction of acoustic loading at lift-off. Use of elaborated technique for multibody launch vehicles with clustered engines and multiduct launch pads is considered.
\end{abstract}

\section{INTRODUCTION}

An acoustical environment generated by a high-temperature supersonic jets acoustical radiation and jet interaction with deflectors is one of principal sources of dynamic loading. Jet interaction with a deflector, in general, is a very complicated gasdynamic problem and there is no reliable theoretical technique for its calculation. Much more it concerns theoretical calculations of acoustical field generated by such interaction. In spite of the considerable progress in computational aeroacoustics calculated data have mainly qualitative nature and hardly applicable for real-world problems [1-4].

The most reliable way to get acoustical field characteristics is to carry out experimental investigations with sufficiently complete simulation of gasdynamic

This is an Open Access article distributed under the terms of the Creative Commons Attribution-Noncommercial License 3.0, which permits unrestricted use, distribution, and reproduction in any noncommercial medium, provided the original work is properly cited. 
and thermodynamic parameters of jet and geometrical parameters of a deflector. But this is itself a very complicated technical and expensive task.

In practice, it is acceptable to use semiempirical techniques based on general theoretical considerations and a generalization of experimental data. Studies of acoustic environment resulting from subsonic and supersonic jets interaction with a deflector have been carried out during many years.

Paper [5-7] contain a thorough review and bibliography on early investigations and include a semiempirical method for predicting acoustic environment resulting from supersonic jet interaction with a deflector based on a model of "free jet deflection." The jet itself (from a nozzle exit to the deflector), jet spreading over the deflector (it has the same characteristics as free jet), and noise reflection from the deflector are considered to be sources of noise generation. But such approach does not always agree with experimental data since, firstly, it does not take into consideration all regions of noise generation on the deflector and, secondly, there is a problem with an assignment of characteristic scales for acoustic sources distributions which represent noise generation regions [6, 8-11].

Another way to find an answer on a problem is to carry out systematic investigation of acoustic fields generated at jet interaction with typical deflectors [12]. Different typical interactions have been studied: an interaction of a supersonic jet with a normal deflector, an interaction of a supersonic jet with single-slope and double-slope deflectors, an interaction of a supersonic jet with a normal deflector having a hole, and so on. In each case, main noise generation regions are selected and corresponding semiempirical acoustic models are created for them. These models use a superposition of contributions from different noise generation regions: a region of undisturbed jet, a region of strong interaction between jet and flow deflector, a region of a jet spreading over deflector surface, solid surfaces as a reflector or a shield for acoustic radiation. The contribution of each source of noise into integral acoustic field varies with a distance between a nozzle and a deflector and with a transverse displacement of a jet. A system of independent acoustical sources with prescribed acoustic power and spectrum of acoustic radiation represents each noise generation region.

Spectral characteristics of resultant acoustical field and overall sound pressure levels are determined by summation of inputs from each source using geometrical acoustics and taking into account possible shielding of some sources by deflector elements. Reflection of noise radiation from deflector elements is calculated by the method of imaginary sources. Acoustic characteristics of the sources are defined based on generalization of manifold experimental data with a use of proper characteristic scales which takes into account jet thermogasdynamic and deflector geometry. Detailed analysis of noise generation regions in the case of jet interaction with a flat plate deflector and inclined deflector is given in [12-15]. Acoustic models for typical interactions can be considered as "bricks," which can be used for estimation of acoustical environment resulting from jet interaction with deflectors of complex shape. Paper [16] deals with making use of such 
approach for estimation of broadband acoustic field generated at interaction of supersonic jets with open from the top scoop-like deflector. In this paper, broadband acoustic field generation at jet interaction with a launch pad having closed long ducts like a tunnel is considered.

\section{PROBLEM STATEMENT}

A scheme of the problem under consideration and reference geometry are shown in Fig. 1 for simple case: a single jet interaction with a single closed duct launch pad. It is assumed that a jet flow together with a mass flow entrained from ambient air enters the launch pad through an opening with diameter $D_{0}$ in a launch table (flat plate) and fills in the duct cross section far enough from a duct exit. Then, due to dissipation processes, a highly nonuniform flow smoothes out and at the exit, there is more or less uniform subsonic flow with average

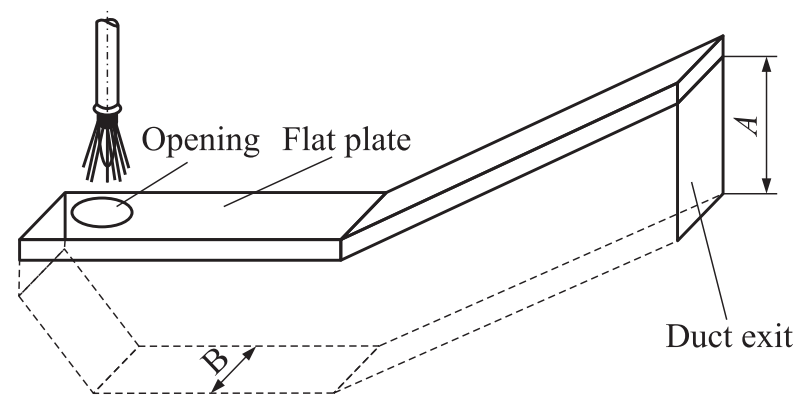

(a)

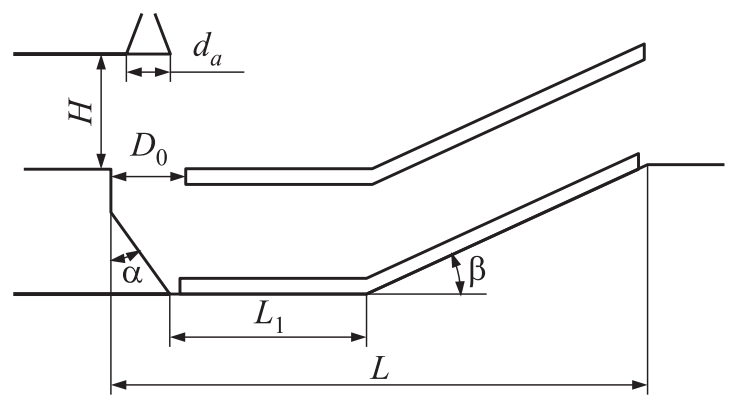

(b)

Figure 1 General view of deflector under consideration 
parameters $u_{3}, \rho_{3}, T_{3}$, and $p_{3}=p_{\infty}$. It is assumed also that a part of a noise generated inside a launch pad is carried away with a flow through the duct exit and, as long as lateral dimension of the jet is less than diameter $D_{0}$ of an opening, there is some acoustic radiation through the launch pad entrance.

Approximately such model of the duct flow is realized for typical main engine jets of launch vehicles when $D / D_{0} \approx 1.0-2.0, D_{0} / d_{a}>2.0$. Here, $D_{0}$ is the entrance opening diameter; $d_{a}$ is the nozzle exit diameter; $D=4 S_{D} / \Pi$ is the effective hydraulic diameter of duct exit; $S_{D}=B A$ is the duct cross-section area ( $B$ is the width and $A$ is the height); and $\Pi=2 A+2 B$ is the duct exit cross-section perimeter.

Analysis shows that the following regions of noise generation can be considered:

- a region of undisturbed jet from nozzle exit up to a launch table (flat plate surface);

- a region of strong interaction between jet and a launch table (flat plate);

- a region of jet noise generation between launch pad entrance and a bottom of the duct;

- a region of noise generation downstream of the duct exit; and

- solid surfaces as reflectors of acoustic radiation.

The interaction of a jet with a launch table (flat plate surface) occurs at such distances from the nozzle exit to the launch table that a lateral dimension of jet in interaction section is more than diameter of an opening in a launch table and/or when there is a transverse displacement of the jet.

The contribution of each region of noise generation in the integral acoustic field varies with a distance between nozzle cut and a launch pad flat plate surface and with a transverse displacement of the jet.

\section{ACOUSTIC MODEL OF JET INTERACTION WITH CLOSED LONG DUCT}

Before analysis of calculated broadband acoustic environment generated at jet interaction with a launch pad having closed long ducts like a tunnel presented in Fig. 1, main assumptions of accepted acoustic model are briefly presented:

- real engine jet is substituted by an isentropically fully expanded equivalent jet (i. e., having the same flow rate and enthalpy); 


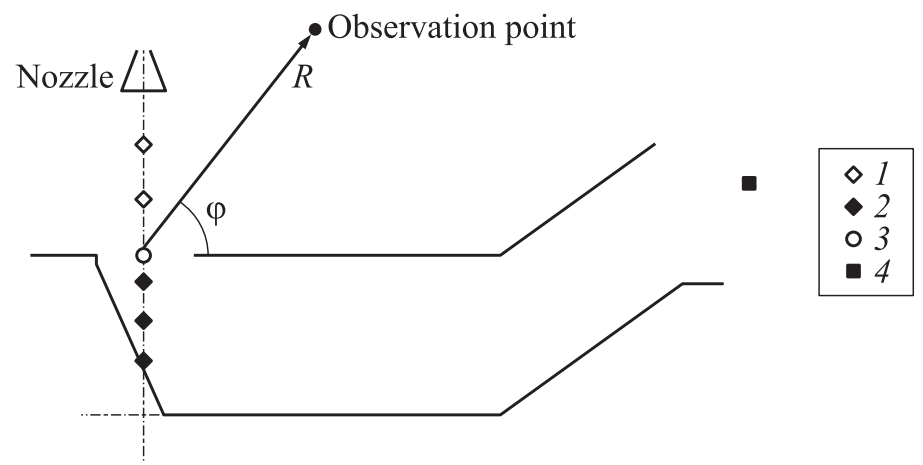

Figure 2 Acoustic sources allocation: 1 - sources representing undisturbed jet above launch table; 2 - sources representing jet noise generation region between launch pad entrance and a bottom of the duct; 3 - a source representing region of strong interaction between jet and a launch table; and 4 - a source representing region of noise generation downstream of the duct exit

- noise generating regions are represented by a system of independent radiating sound sources with prescribed sound power, spectrum, and directivity (Fig. 2);

- spectral characteristics of resultant sound field and overall sound pressure levels are determined by summation of inputs from every source, using geometrical acoustics;

- acoustic power, radiation spectrum, and directivity factor of sources are defined from a generalization of numerous experimental data for interacting jets, using scales which take into account the jet aerothermodynamics; and

- region of noise generation downstream of the duct exit (subsonic jet-like flow) is represented by a single radiating source with prescribed location, acoustic power (a sum of subsonic flow acoustic power and acoustic power of noise generated in launch pad), and spectrum of acoustic radiation.

\subsection{Jet Gasdynamic Model}

Real engine jet is substituted by an isentropically fully expanded equivalent jet, having the same flow rate and enthalpy. Aerothermodynamic parameters of this equivalent jet are calculated by semiempirical model described in $[10,11]$. The basic idea of jet aerodynamic model is use of empirical similarity law for axial velocity distribution in the main region of supersonic isobaric jet: 


$$
\frac{u_{m}}{u_{j}}=1-\exp \left(\frac{1.4}{1-2 \xi}\right) ; \quad \xi=\frac{x}{x_{m}}
$$

where $u_{m}$ is the velocity at the axis of fully expanded equivalent jet; $u_{j}$ is the nozzle exit velocity of fully expanded equivalent jet; $x$ is the distance along jet axis; and $x_{m}$ is the distance from nozzle exit to cross section where $u_{m}=0.75 u_{j}$. The quantity $x_{m}$ can be calculated from experimental relation:

$$
\frac{x_{m}}{D_{j}}=6\left[1+\frac{\mathrm{M}_{j}^{2}\left(\gamma_{j}-1\right)}{2}\right]\left(1+\frac{I_{0}}{2}\right)-\frac{\mathrm{M}_{j}^{2}\left(\gamma_{j}-1\right)}{3}
$$

where $I_{0}$ represents the ratio of ambient enthalpy to total enthalpy of the jet; $D_{j}$ is the exit diameter of fully expanded jet; $\mathrm{M}_{j}$ is the exit Mach number; and $\gamma_{j}$ is the specific heat ratio of fully expanded jet. Distance $x_{m}$ corresponds to the location of the turbulence peak and to the maximum of axial velocity gradient in the jet. For acoustic model, it is assumed that the turbulence peak location coincides with the sound power peak location in the jet. This quantity is used as a linear characteristic scale, which defines both the jet thermogasdynamic parameter distributions and sound power distribution for region of undisturbed jet and jet under the launch table.

\subsection{Acoustic Model for Undisturbed Portion of Jet and Jet Portion Between Launch Pad Entrance and Bottom of the Duct}

The sound power density distribution normalized by the overall acoustic power of free jet for undisturbed portion of jet and jet portion between launch pad entrance and a bottom of the duct is set by relation

$$
d\left(\frac{W_{a}}{W_{\Sigma a}}\right)=F(\xi) d \xi
$$

where $W_{a}(\xi)$ is the acoustic power radiated by jet region from the nozzle exit to cross section $\xi ; W_{a \Sigma}$ is the integral acoustic power radiated by free jet (if there is no deflector); and

$$
F(\xi)= \begin{cases}0.003 & \text { at } 0 \leq \xi<0.075 \\ 1.04 \xi-0.075 & \text { at } 0.075 \leq \xi<0.65 \\ 0.6 & \text { at } 0.65 \leq \xi<1.24 \\ -0.29 \xi+0.96 & \text { at } 1.24 \leq \xi \leq 2.293 \\ 0 & \text { at } \xi>2.293\end{cases}
$$

Maximum value of density locates around $\xi=1$ that is at location of the turbulence peak and maximum of axial velocity gradient in the jet. The integral 


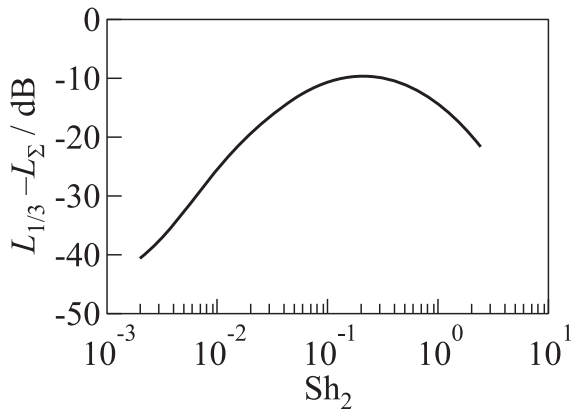

(a)

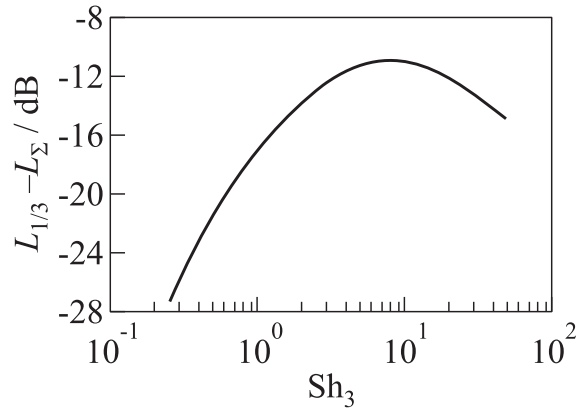

(b)

Figure 3 Normalized one third octave spectra of acoustic pressure induced in outside acoustic field by acoustic sources representing jet $(a)$ and by the source at duct exit $(b)$

acoustic power of free jet is calculated through the free jet acoustic efficiency $\eta$ which is set by empirical dependence on $u_{j}$ presented in [16].

Overall sound pressure level (OASPL) $L_{\Sigma i}$ induced in outside acoustic field by each acoustic source representing undisturbed jet and jet portion between launch pad entrance and a bottom of the duct is determined through known sound power level of each source $L_{W i}$ by relation

$$
L_{\Sigma i}=L_{W i}=101 g \Omega_{i} R_{i}
$$

where $R_{i}$ is the distance from the $i$ th source to observation point; and $\Omega_{i}$ is the solid angle of radiation for the $i$ th source which depends on launch pad geometry.

One third octave spectra of acoustic pressure $L_{1 / 3}(f)$ induced in outside acoustic field by each acoustic source representing undisturbed jet and jet portion between launch pad entrance and a bottom of the duct is specified by empirical dependence $L_{1 / 3}-L_{\Sigma}=F\left(\mathrm{Sh}_{2}\right)$ shown in Fig. $3 a$. Here, $\mathrm{Sh}_{1}=\mathrm{Sh} / \mathrm{Sh}_{\max }$ is the modified Strouhal number, which takes into account the distribution of characteristic frequency of sources (frequency of a maximum in spectrum generated by each source $f_{\max }$ ) along jets:

$$
\mathrm{Sh}=\frac{f D_{j}}{u_{j}} ; \quad \mathrm{Sh}_{\max }=\frac{f_{\max } D_{j}}{u_{j}}=\frac{K\left(T_{j}, \varphi, x_{m}\right)}{\xi} .
$$

It is necessary to note that when there is a transverse displacement of the jet acoustic power of sources in jet region between launch pad entrance and a bottom of the duct, $W_{a}^{\prime}$ must be reduced in comparison with acoustic power of corresponding sources in free jet $W_{a}$, namely:

$$
W_{a}^{\prime}=k W_{a} ; k=\left(1-\frac{F_{w}}{F_{j}}\right)^{1.35}
$$


where $F_{w}$ is the part of jet impulse which interacts with launch table; and $F_{j}$ is the jet impulse at the exit of effective fully expanded nozzle.

\subsection{Acoustic Model for Region of Strong Interaction Between Jet and Launch Table (Flat Plate)}

As already mentioned, the interaction of a jet with a launch table (flat plate surface) occurs at distances from the nozzle exit to the launch table when a lateral dimension of jet in interaction section is more than diameter of an opening in a launch table and/or when there is a transverse displacement of the jet. Acoustical model for such interaction is presented in $[12,16]$.

\subsection{Acoustic Model for Noise Generation Downstream of the Duct Exit}

It is assumed that the region of noise generation downstream of the duct exit (subsonic jet-like flow) is represented by a single radiating source with prescribed location, acoustic power, and spectrum of acoustic radiation (see Fig. 2). Analysis of available experimental data has shown that characteristics of this radiating source are as follows.

Overall sound pressure level $L_{\Sigma}$ created at given point of observation is determined through known acoustic power of the source by formula:

$$
l_{\Sigma}=10 \lg \left(\frac{W_{a}^{D}}{W_{0}}\right)-10 \lg 2 \pi R_{D}^{2}
$$

where $W_{a}^{D}$ is the acoustic power of the source; $W_{0}$ is the threshold power, $W_{0}$ $=10^{-12} \mathrm{~W}$; and $R_{D}$ is the distance from the source to observation point with coordinates.

Acoustic power of the source $W_{a}^{D}$ is determined by relation:

$$
W_{a}^{D}=\eta_{1} \frac{\rho_{3} u_{3}^{8} D^{2}}{c_{\infty}^{5}}+\eta_{2} W_{a D} .
$$

Here, $D=4 S_{D} / \Pi ; S_{D}=B A$ is the duct exit cross-section area; $\Pi=2 A+2 B$ is the duct exit cross-section perimeter; $\rho_{3}$ is the average flow density at duct exit; $u_{3}$ is the average flow velocity at duct exit; $\eta_{1}=2.0 \cdot 10^{-4}$ is the acoustic efficiency of subsonic jet; $W_{a D}=W_{a \Sigma}-W_{a}\left(\xi_{H}\right)$ is the acoustic power radiated by a region of free jet from nozzle exit up to the launch pad deflector; $\xi_{H}=H / x_{m}$; and $\eta_{2}=0.05$.

Spectrum of sound pressure radiated by the source at duct exit in outside acoustic field is represented by the dependence $L_{1 / 3}-L_{\Sigma}=F\left(\mathrm{Sh}_{3}\right)$ shown in 
Fig. $3 b$ where $L_{1 / 3}$ is the $1 / 3$-octave spectral level of sound pressure induced by the source at a point of outside acoustic field; $L_{\Sigma}$ is the OASPL induced by the source at a point of outside acoustic field; and $\mathrm{Sh}=\left(f D / u_{3}\right)$ is the Strouhal number.

Average flow parameters in the exit cross section of the duct (with index " 3 ") are determined from average flow parameters at the duct entrance opening (with index "1") by equality of calculated with average parameters flow rate and total enthalpy in these sections. At this, it is supposed that static pressure at exit cross section of the duct is equal to ambient pressure. The following relation can be written:

$$
\begin{gathered}
\frac{u_{3}}{u_{1}}=\frac{\mathrm{M}_{3}}{\mathrm{M}_{1}} \sqrt{\frac{1+\mathrm{M}_{1}^{2}\left(\gamma_{1}-1\right) / 2}{1+\mathrm{M}_{3}^{2}\left(\gamma_{1}-1\right) / 2}}, \quad \frac{\rho_{3}}{\rho_{1}}=\frac{1+\mathrm{M}_{3}^{2}\left(\gamma_{1}-1\right) / 2}{1+\mathrm{M}_{1}^{2}\left(\gamma_{1}-1\right) / 2} ; \\
\mathrm{M}_{3}^{2}\left(1+\frac{\gamma_{1}-1}{2} \mathrm{M}_{3}^{2}\right)=\mathrm{M}_{1}^{2}\left(1+\frac{\gamma_{1}-1}{2} \mathrm{M}_{1}^{2}\right)\left(\frac{D_{0}}{D}\right)^{4} .
\end{gathered}
$$

If one introduces $\rho_{1} u_{1}^{2} S_{1}=G_{1}$ and $\rho_{1} u_{1}^{2} S_{1}=F_{1}\left(G_{1}\right.$ and $F_{1}$ are the flow rate and impulse at the duct entrance opening, respectively; and $S_{1}$ is the opening area), then $u_{1}=F_{1} / G_{1}$. Denote $\theta=u_{1} / u_{j}=F_{1} / u_{j} G_{1}$ ( $u_{j}$ is the velocity at the exit of effective fully expanded nozzle which substituting real nozzle), then for thermodynamic parameters of average flow at the opening, one has:

$$
C_{p 1}=\theta C_{p j}+(1-\theta) C_{p \infty} ; \quad R_{1}=\theta R_{j}+(1-\theta) R_{\infty} ; \quad \gamma_{1}=\frac{1}{1-R_{1} / C_{p 1}} .
$$

From flow rate and impulse conservation, it follows:

$$
\mathrm{M}_{1}=\mathrm{M}_{j}\left(\frac{\gamma_{j} F_{1} D_{j}}{\gamma_{1} F_{j} D_{0}}\right)^{0.5} ; \quad \rho_{1}=\rho_{j}\left(\frac{G_{1} D_{j}}{G_{j} D_{0}}\right)^{2} \frac{F_{1}}{F_{j}}
$$

where $D_{j}$ is the diameter of nozzle exit for effective fully expanded nozzle which substitutes real nozzle. In the above relations, indices " $j$ " and " $\infty$ " relate to jet parameters at the exit of the equivalent nozzle and ambient air parameters, respectively.

Determination of flow rate $G_{1}$ and impulse $F_{1}$ at the duct entrance opening can be done, for example, with a use of simplified aerodynamic model of supersonic fully expanded jet presented in [8] in the following way.

At first, a distance $X^{*}$ from nozzle exit to the jet cross section where external boundary of the jet $r_{2}$ is equal to the radius of the opening in the launch table $D_{0} / 2$ can be found (the jet lock in the opening in launch table in that case). Normalized total flow rate at this jet cross section $G_{*}$ can be calculated by relation:

$$
G_{*}=\frac{G\left(r_{2}\right)}{G_{j}}=\frac{2 \rho_{m} u_{m}}{\rho_{j} u_{j} r_{j}^{2}} \int_{0}^{D_{0} / 2} \frac{\rho u}{\rho_{m} u_{m}} r d r .
$$


It is supposed ( $H$ is the distance between equivalent nozzle exit and launch table; $G_{j}$ is the flow rate at the exit of effective fully expanded nozzle; and $F_{j}$ is the jet impulse at the exit of effective fully expanded nozzle):

- if $H \leq X^{*}$, then $G_{1}=G_{*} \times G_{j}, F_{1}=F_{j}$;

- if $H>X^{*}$, then

$$
\begin{aligned}
G_{D_{0}} & =\frac{G\left(r=D_{0} / 2\right)}{G_{j}}=\frac{2 \rho_{m} u_{m}}{\rho_{j} u_{j} r_{j}^{2}} \int_{0}^{D_{0} / 2} \frac{\rho u}{\rho_{m} u_{m}} r d r \\
G_{1} & =G_{D_{0}} \times G_{j} ; \quad F_{1}=F_{j}-F_{w}
\end{aligned}
$$

where $F_{w}$ is the part of jet impulse which interacts with launch table.

\subsection{Resultant Sound Field}

Spectral characteristics of resultant acoustical field and overall sound pressure levels at given point of observation are determined by summation of inputs from each source using geometrical acoustics. Reflection of noise radiated by undisturbed region of jet above the launch table from ground surface is calculated using a method of imaginary sources. Ground surface is assumed to be absolutely reflecting. An imaginary source located as mirror image to reflecting plane possessed the same acoustic power and radiated spectrum is assigned to each source which is used for calculations of acoustic field generated by undisturbed region of the jet above the launch table.

\section{MULTINOZZLE AND MULTIDUCT CONFIGURATIONS}

The approach formulated in the previous section can be easily extended to the case of multinozzle configuration and to the case of launch pad with several ducts.

Noise generated at jet interaction with closed long duct consists of two parts. First part is a noise radiated by the region of undisturbed jet from nozzle exit up to a launch table, a region of strong interaction between jet and a launch table (flat plate), a region of jet noise generation between launch pad entrance and a bottom of the duct. By convention, this part of total noise can be named as noise at duct entrance. Second part is the noise from a region of noise generation downstream of the duct exit. 
As for the first part of noise, when there is a system of noninteracting jets, calculation of acoustic field characteristics for each jet is carried out in series with subsequent summation of inputs in the given point of observation. If there is a system of interacting jets (compact assembly), it can be substituted by equivalent effective single jet having the same flow rate, flow impulse, and excess total enthalpy flow as a sum of corresponding parameters for real nozzles. Details of such substitution are discussed in $[12,16]$.

Regarding the region of noise generation downstream of the duct exit, a number of radiating sources is equal to the number of ducts. Besides, it is necessary to set a distribution of incoming jets between ducts.

For calculation of averaged flow parameters at duct (or ducts) exit, a system of real engine nozzles is substituted by a single (effective) equivalent nozzle having the same flow rate, flow impulse, and excess total enthalpy flow as a sum of corresponding parameters for real nozzles. At this, at the exit, aggregate effective hydraulic diameter of exit is used, which is determined through effective hydraulic diameters of each duct exit.

A substitution of a system of real engine nozzles (in general case, possessing different nozzle exit diameters and different values of flow parameters at nozzle exit) by a single equivalent nozzle having the same flow rate, flow impulse, and excess total enthalpy flow as a sum of corresponding parameters for real nozzles is convenient to perform in two stages. At first, it is necessary to substitute each real jet by an isentropically fully expanded equivalent jet, having the same flow rate and enthalpy, and then, this system of isentropically fully expanded nozzles is substituted by a single equivalent nozzle having the same flow rate, flow impulse, and excess total enthalpy flow as a sum of corresponding parameters for isentropically fully expanded nozzles. Following system of algebraic equations should be solved:

$$
\begin{gathered}
\rho_{\text {eq }} u_{\text {eq }} S_{\text {eq }}=\sum_{i=1}^{N} \rho_{i j} u_{i j} S_{i j} ; \quad \rho_{\text {eq }} u_{\text {eq }}^{2} S_{\text {eq }}=\sum_{i=1}^{N} \rho_{i j} u_{i j}^{2} S_{i j} ; \\
\rho_{\text {eq }} u_{\text {eq }}\left(H_{\text {eq }}-H_{\infty}\right) S_{\text {eq }}=\sum_{i=1}^{N} \rho_{i j} u_{i j}\left(H_{i j}-H_{\infty}\right) S_{i j} ; \\
C_{p \text { eq }}=\sum_{i=1}^{N} C_{p a i} \frac{G_{i}}{G_{\text {eq }}} ; \quad R_{\text {eq }}=\sum_{i=1}^{N} R_{a i} \frac{G_{i}}{G_{\text {eq }}}, \quad \gamma_{\text {eq }}=\frac{1}{1-R_{\text {eq }} / C_{p \text { eq }}} .
\end{gathered}
$$

Here, index "eq" refer to the parameters at equivalent nozzle exit, index " $i j$ " to the parameters at isentropically fully expanded nozzle exits, $i=1,2, \ldots, N$ (number of nozzles); and $G$ is the flow rate.

In the simplest case, when there are $N$ nozzles with the same parameters at nozzle exit, one has a simple relation for diameter of equivalent nozzle:

$$
D_{\text {eq }}=D_{a} \sqrt{N} \text {. }
$$


In general case, it is necessary to solve the above system of algebraic equations.

\section{RESULTS OF CALCULATIONS AND THEIR VALIDATION}

For validation of set forth acoustic model, ad hoc experimental investigations of acoustic field characteristics have been done at supersonic jet interaction with launch pad model of the shape presented in Fig. 1. Geometrical parameters of the launch pad were as follows: $D_{0} / d_{a}=2.53 ; L / d_{a}=16.8 ; L_{1} / d_{a}=7.0$; $A / d_{a}=7.64 ; B / d_{a}=3.83 ; \alpha=30^{\circ}$; and $\beta=30^{\circ}$.

Jet exhaust was created by burning of a charge of solid propellant within a gas generator. Gas generator of cone-cylinder shape with contoured nozzle was mounted on special device which lets to set different distances $H$ between nozzle exit and launch pad. In these tests, jet parameters at the nozzle exit were: Mach number $\mathrm{M}_{a}=3.57$, stagnation temperature $T_{0 a}=2280 \mathrm{~K}$, ratio of static pressure at nozzle exit to ambient pressure $n=0.6$, specific heats ratio $\gamma=1.22$, and gas constant $R_{a}=345 \mathrm{~J} /(\mathrm{kg} \cdot \mathrm{deg})$.

Acoustic pressure measurements were conducted in a free acoustic field and on a surface of gas generator body in the symmetry plane of launch pad.

Calculated (lines) one third octave spectra of acoustic pressure $L_{1 / 3}(f)$ in several points of measurements (p.1, p.2, p.3, p.4) for two distances $H$ between nozzle exit and launch pad are compared in Fig. 4 with test data (symbols). Polar coordinates of measurement points (see Fig. 2):

$$
\begin{aligned}
& \text { p. } 1-R / d_{a}=19.8, \varphi=35.5^{\circ} \text {; } \\
& \text { p. } 2-R / d_{a}=30.0, \varphi=77.5^{\circ} \text {; } \\
& \text { p. } 3-R / d_{a}=19.4, \varphi=88^{\circ} \text { at } H / d_{a}=0 \text { and } R / d_{a}=32.9, \varphi=89^{\circ} \text { at } \\
& \quad H / d_{a}=13.5 ; \text { and } \\
& \text { p. } 4-R / d_{a}=1.5, \varphi=63^{\circ} \text { at } H / d_{a}=0 \text { and } R / d_{a}=14.9, \varphi=87.5^{\circ} \text { at } \\
& \quad H / d_{a}=13.5 \text {. }
\end{aligned}
$$

Presented calculated data include both total values (denoted as $\Sigma$ ) and inputs:

- from noise radiated $\left(\sum\right)$ by a region of undisturbed jet from nozzle exit up to a launch table, a region of strong interaction between jet and a launch table (flat plate), a region of jet noise generation between launch pad entrance and a bottom of the duct (noise at duct entrance, denoted as $I)$; and

- from noise generation region downstream of the duct exit (denoted as $I I$ ). 

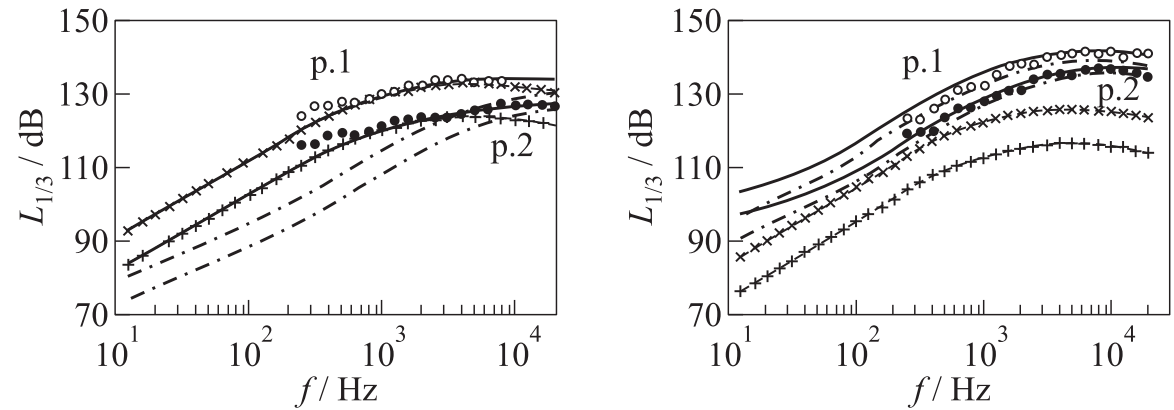

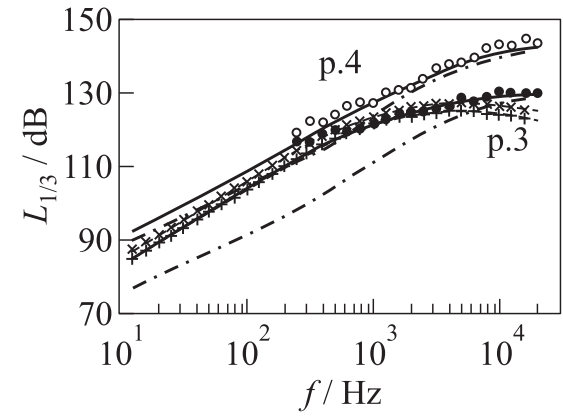

(a)

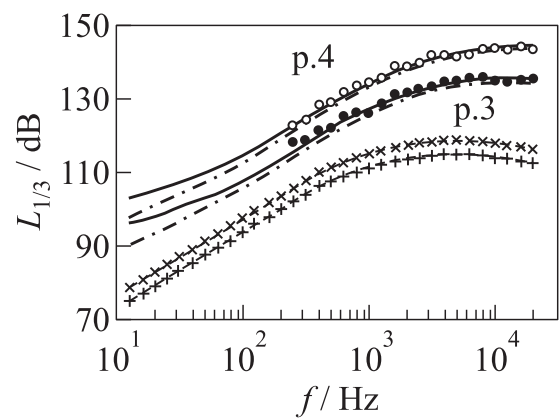

(b)

Figure 4 Calculated and measured one third octave spectra of acoustic pressure at different points in outside acoustic field $\left(1-\sum ; 2-I\right.$; and $\left.3-I I\right):(a) H / d_{a}=0$; and $(b) H / d_{a}=13.5$

The data show that input of noise generation region downstream of the duct exit into acoustic field is considerable only at $H=0$ and at points near duct exit exceeds input of noise at duct entrance. With $H$ increasing, the input of noise generation region downstream of the duct exit decreases and at $H / d_{a}=13.5$, the input of noise at duct entrance predominates. In a whole, an agreement of calculated and experimental data is good.

Variations of calculated and measured OASPL along gas generator body ( $l$ is the a distance from

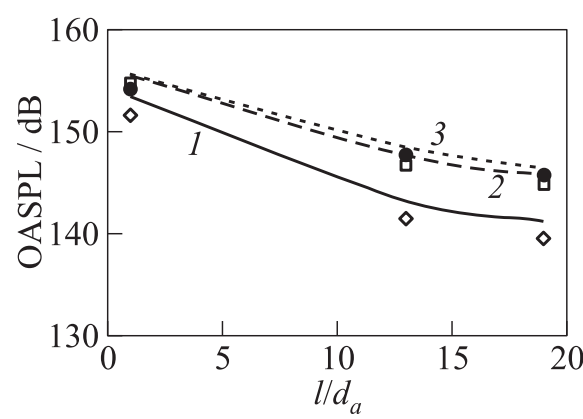

Figure 5 Variation of calculated (lines) and measured (symbols) OASPL along gas generator body $\left(1-H / d_{a}=0 ; 2-8.75\right.$; and $\left.3-H / d_{a}=13.5\right)$ 
nozzle exit) at different distances $H$ between nozzle exit and launch pad are shown in Fig. 5. As distinct from calculated and experimental data for open launch pad presented in [16], maximal acoustic loading occurs at some distance between nozzle exit and launch pad.

Comparison of acoustic pressure spectra generated at interaction of the same jet with launch pads having the same configuration but differing from each other by presence or absence of upper cover shows that for closed duct at small distances between nozzle exit and launch pad, intensity of acoustic field is considerably lower (Fig. 6).

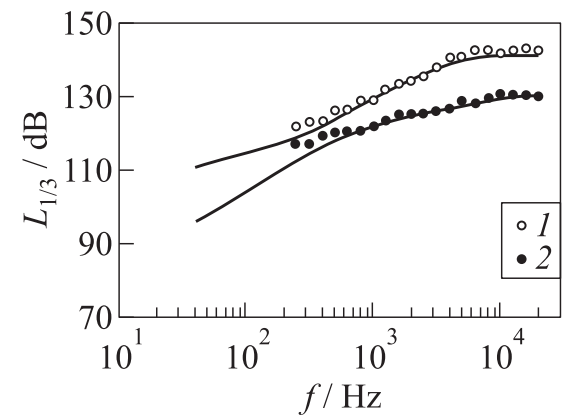

(a)

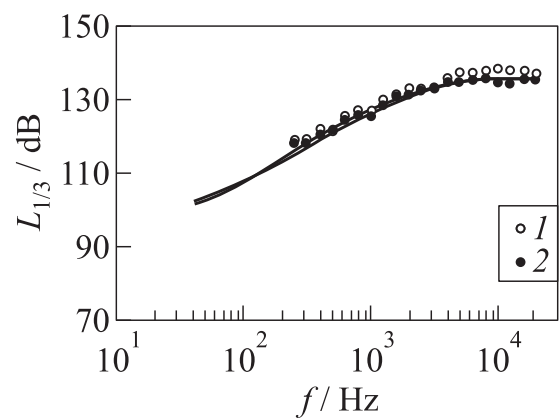

(b)

Figure 6 Calculated (lines) and measured (symbols) one third octave spectra of acoustic pressure for open (1) [16] and closed (2) ducts for different distance $H$ at point p.3: (a) $H / d_{a}=0$; and $(b) H / d_{a}=13.5$

\section{CONCLUDING REMARKS}

Semiempirical acoustic model assigned for estimation of characteristics of broadband acoustic field generated at interaction of supersonic high-temperature jet with a launch pad having closed long ducts like a tunnel is elaborated.

The acoustic model uses a superposition of contributions from different noise generation regions. Noise generation regions are represented by a system of independent acoustic sources with prescribed acoustic power and spectrum of acoustic radiation. Acoustic characteristics of the sources are defined based on generalization of manifold experimental data with the use of proper characteristic scales which takes into account jet thermogasdynamic and deflector geometry.

Comparisons of calculated results with experimental data show that the elaborated acoustic model allows to make reliable estimations of acoustical field characteristics as a function of deflector geometry and jet parameters, to study inputs 
from different regions of noise generation and to analyze the means for reduction of acoustic loading at launch vehicles liftoff.

\section{ACKNOWLEDGMENTS}

This work has been supported by the Russian Foundation for Basic Research (project 07-08-00180).

\section{REFERENCES}

1. Kandula, M. 2004. Numerical simulation of noise from a supersonic jet passing through a rigid duct. AIAA Paper No. 2004-2935.

2. Kawai, S., R. Takaki, S. Tsutsumi, and K. Fjii. 2007. Computational aeroacoustic analysis of overexpanded supersonic jet impingement on a flat plate with/without hole. FEDSM2007 Proceedings: 5th Joint ASME/JSME Fluids Engineering Conference. San Diego, USA. FEDSM2007-37563.

3. Tsutsumi, S., R. Takaki, T. Shima, K. Fujii, and M. Arita. 2008. Generation and propagation of pressure waves from H-IIA launch vehicle at lift-off. AIAA Paper No. 2008-390.

4. Tsutsumi, S., S. Kato, K. Fukuda, R. Takaki, and K. Ui. 2009. Effect of deflector shape on acoustic field of launch vehicle at lift-off. AIAA Paper No. 2009-328.

5. Eldred, K. M. 1971. Acoustic loads generated by the propulsion system. NASA SP-8072.

6. Varnier, J., W. Raguenet, and D. Gely. 1998. Noise radiated from free and impinging hot supersonic jets. AIAA Paper No. 98-2206.

7. Crocker, M. J. 2008. Noise of rocket exhaust at the launch of a spacecraft. Workshop (International) on Acoustics and Vibration in Egypt (IWAVE 2008). Faculty of Engineering, Ain Shams University.

8. Varnier, J., and W. Raguenet. 2002. Experimental characterization of the sound power radiated by impinging supersonic jets. AIAA J. 40(5).

9. Varnier, J. 2001. Experimental study and simulation of rocket engine free jet noise. AIAA J. 39(10).

10. Koudriavtsev, V.V., J. Varnier, and A. V. Safronov. 2004. A simplified model of jet aerodynamics and acoustics. AIAA Paper No. 2004-2877,

11. Kudryavtsev, V.V., and A.V. Safronov. 2008. Approximate model for prediction of the acoustic field induced by high-temperature supersonic jets. 14th Conference (International) on the Methods of Aerophysical Research (ICMAR 2008) Proceedings. ISBN 978-5-98901-040-0 (CD-ROM). Novosibirsk-Moscow, Russia.

12. Dementjev, V., V. Koudriavtsev, S. Rybak, et al. 1999. Technique for predicting the acoustical environment resulting from a launch vehicle engine jets interaction with a launch pad. 1st European Conference on Launcher Technology Proceedings. Launch Vehicle Vibrations. Toulouse, France. 
13. Koudriavtsev, V.V. 2000. Acoustic environment at jet interaction with a plate. 29th Congress (International) and Exhibition on Noise Control Engineering (INTERNOISE 2000) Proceedings. Nice, France.

14. Koudriavtsev, V.V., and A.V. Safronov. 2001. Noise generation at supersonic jet interaction with inclined deflector. Congress (International) and Exhibition on Noise Control Engineering (INTERNOISE 2001) Proceedings. The Hague, The Netherlands.

15. Kudryavtsev, V.V., and A. V. Safronov. 2007. Noise at supersonic jet interaction with a deflector. West-East High Speed Flow Field Conference Proceedings. CDROM. Moscow, Russia.

16. Kudryavtsev, V.V., and A. V. Safronov. 2008. Acoustic model for supersonic jet interaction with a complex deflector. 6th European Symposium on Aerothermodynamics for Space Vehicles Proceedings. Versailles, France: ESA. SP-659. 